\title{
Reproductive medicine 2014: the year in review
}

\author{
David F. Albertini
}

Published online: 22 November 2014

(C) Springer Science+Business Media New York 2014

2014 has been significant year for JARG as more and more of our colleagues working in the ART and reproductive genetics areas are coming to realize our contributions to the practice and science behind reproductive medicine. This month alone we feature an up-to-date review on the genetics of Primary Ovarian Insufficiency by Labarta and colleagues continuing our series of reviews emphasizing the technological and academic foundations resulting from molecular genetics as we know it today. For some subjects frequenting the pages of JARG in 2014, the history of specialty areas such as fertility preservation are relatively speaking in their adolescence. In contrast, those stemming from the continued use of cryotechnology in human ARTs have roots spanning decades of investigation. To wit, it has been 65 years since the landmark publication of Polge and Hammond in Nature on the application of vitrification for the storage of equine sperm, and our readership will find this month a series of reports exemplifying the continued efforts of our colleagues as they refine and modify this technology for the benefit of infertility patients. While technologies as seasoned as vitrification will remain a mainstay in the practice of gamete, embryo, and tissue cryopreservation, evolving as needed, many of the more "youthful" items on our ART menus today will continue to flux in and out of style pending changes in the landscapes being etched by the "omics" generation.

JARG remains committed to serving the patients, scientists, and clinicians involved with human ARTs by educating our readership with coverage of the latest breakthroughs in

Capsule From taking control of stem cell pluripotency, to defining the operational partners in fertilization, to making inroads into the mechanisms underlying PCOS, 2014 has been a fruitful year of discovery in basic science with immediate and long-term implications for reproductive health.

D. F. Albertini $(\bowtie)$

University of Kansas Medical Center, Kansas, KS, USA

e-mail: dalbertini@kumc.edu science and technology that for better or worse bring some sense of satisfaction to the reproductive medicine community on an international scale. With this commitment in mind, we bring you for the second year in a row now our top seven selections of original contributions to the literature with the likelihood of changing the way we think about human reproduction broadly, and treatment of infertility specifically.

Once again, several significant advances have appeared in the past year that take their origins from the powerful genetic manipulations scientists are able to conduct in mice seeking answers to many of the most fundamental questions in reproductive biology. Among these was the study Yamauchi and colleagues from the University of Hawaii showing that two genes alone residing on the $\mathrm{Y}$ chromosome are sufficient to determine sex and support spermatogenesis in what may presage the identification of genetic and epigenetic factors contributing to male infertility [1].

At the other end of the reproductive axis comes an intriguing case of technological bravado bound to raise eyebrows in the human ART community once the relevance of these studies in mice reach a point of "translatability." Reproductive endocrinologists take for granted, and as a patient-specific daily challenge, manipulating gonadotropin levels and their ovarian output as the very framework upon which $\mathrm{COH}$ and egg retrieval set into action the cascade of events used to treat infertility. From the laboratory of T. Raj Kumar (who happens to be one of our Senior Editors) a study appeared which takes genetic manipulations in mice to a new level in teasing apart the biochemistry of LH and FSH secretion from the pituitary that ultimately determines whether the gonads perceive pulsatile or constitutive patterns of stimulation [2]. While these findings are "pregnant" with evolutionary implications, this research could have a direct bearing on how patients with poor responsiveness might be managed in a more effective fashion.

On the stem cell front, more of the molecular underpinnings that establish and maintain pluripotency have been 
unearthed to add to the complement of histone modifications that were all the rage only a year ago. So along comes citrullination by a family of enzymes known as peptidylarginine deiminases, or PADIs for short, that have been shown to regulate the state of chromatin compaction required to activate transcriptional networks important to the pluripotent condition in stem cells but also in mouse embryos, adding to the list chromatin modifications that may at some point play into embryo selection [3].

As we featured in JARG earlier this year, characterizing the gender-specific molecular partners that mediate fertilization continues to be a matter of controversy, with many hopeful candidates coming and going over the years. But a fascinating and telling series of experiments by Bianchi and colleagues in Cambridge, England, has lead to the identification of a receptor ligand pair that have passed the test in mouse genetic experiments and appear to carry on their courtship in other mammals including humans [4]. Even more interesting is the fact that the egg-born receptor for sperm doubles as a folate receptor (Folr4) once again reminding us that while we would like each gene product to carry with it a singular function, for many an essential molecule in reproduction doing double or triple duty may have become the byproduct of selective pressure assuring fecundity. And stay tuned because the rapid release of Juno from the oolemma is suspected to be involved in the block to polyspermy and as we will soon see raises the mounting prospect that exosomes, one of the latest intercellular signaling devices capturing attention among many disciplines, may be deployed in many an event mediating reproduction.

Among the exciting accoutrements brought along in the exosome feeding frenzy is the fact that they exist in bodily fluids of various kinds-urine, blood, even follicular fluid! And a study by Jan McAllister and her colleagues who have been in the polycystic ovary syndrome clan for many years now provides evidence for the presence of an exosomal biomarker that could improve disease diagnosis [5]. These studies go on to show that not only is the RNA for DENND1A detectable in the urine of PCOS women, the gene product itself exerts potent effects on androgen biosynthesis in normal thecal cells causing them to assume a PCOS phenotype. And speaking of androgens, an equally enticing piece of the PCOS puzzle was also provided this year in a paper by Sen and colleagues [6]. Teasing apart the mechanisms behind regulating follicular steroidogenesis has been a challenge for many years now, and this study places into perspective a rational framework that could readily provide insights into origins and treatment of PCOS and the role that androgens play systemically in the reproductive health of men and women.

And finally, the long-awaited confirmation of low-cost IVF as a safe and efficient way to produce live offspring has been realized. As reported in RBM Online, Van Blerkom and colleagues have produced the first babies born of a much simplified approach to IVF [7]. So as not to debase, devalue, or deconstruct the societal and medical impact traditional human ARTs has had since its inception in 1978, efforts such as these open new avenues for the delivery of care to those patients desperately in need of treatment but whose geographic or economic situations prevent them from doing so. Offering the best care possible to as many who desire it remains our priority.

\section{References}

1. Yasuhiro Y, Riel JM, Zoia S, Monika A. Monika A. WardTwo Y Genes Can Replace the Entire Y Chromosome for Assisted Reproduction in the Mouse. Science. 2014; 343:69-71. doi:10.1126/ science 1242544.

2. Wang $\mathrm{H}$, et al. Redirecting intracellular trafficking and the secretion pattern of FSH dramatically enhances ovarian function in mice. Proc Natl Acad Sci U S A. 2014; 111:5735-40. doi:10.1073/pnas. 1321404111.

3. Christophorou, et al. Citrullination regulates pluripotency and histone H1 binding to chromatin. Nature. 2014. doi:10.1038/nature12942.

4. Bianchi, et al. Juno is the egg Izumo receptor and is essential for mammalian fertilization. Nature. 2014. doi:10.1038/nature13203.

5. McAllister, et al. Overexpression of a DENND1A isoform produces a polycystic ovary syndrome theca phenotype. Proc Natl Acad Sci U S A. 2014. doi: $10.1073 /$ pnas. 1400574111 .

6. Sen, et al. Androgens regulate ovarian follicular development by increasing follicle stimulating hormone receptor and microRNA125b expression. Proc Natl Acad Sci U S A. 2014; 111:3008-13. doi:10.1073/pnas. 1318978111.

7. Van Blerkom J, et al. First births with a simplified culture system for clinical IVF and embryo transfer. Reprod Biomed Online. 2014; 28 : $310-20$. 